\title{
A CATEGORY THEOREM FOR TSUJI FUNCTIONS
}

\author{
PETER COLWELL
}

ABSTRACT. If $H$ denotes the functions analytic in the open unit disk with the topology of uniform convergence on compact subsets, both the Tsuji functions in $H$ and the functions in $H$ with nonempty Tsuji sets comprise sets of first category in $H$. A question is posed about the category of a class of functions containing the Tsuji furctions.

1. Introduction. Let $D=\{|z|<1\}, C=\{|z|<1\}$, and $H$ be the collection of functions analytic in $D$ with the topology of uniform convergence on compact subsets of $D$. For each $f \in H$ and $z \in D$ let $f^{*}(z)=\left|f^{\prime}(z)\right| /\left(1+|f(z)|^{2}\right)$, the spherical derivative of $f$ at $z$. For each $r, 0<r<1$, and each $f \in H$, we let $L(f, r)=\int_{0}^{2 \pi} r f^{*}\left(r e^{i \phi}\right) d \phi$. If $\lim \sup _{r \rightarrow 1^{-}} L(f, r)<\infty, f$ is called a Tsuji function. (First introduced in [4], the Tsuji functions have since been extensively studied [1], [2], [3].)

If, for each $\alpha \in D$, we let $\phi_{\alpha}(z)=(z-\alpha) /(1-\bar{\alpha} z)$, the Tsuji set of $f \in H$ is the set of points $\alpha \in H$ for which $f \circ \phi_{\alpha}$ is a Tsuji function. Tsuji sets were defined in [2], and they have not yet been characterized. In this note we prove the following

Theorem. The collection of functions in $H$ which have a nonempty $T s u j i$ set is of first category in $H$.

This result also shows that the Tsuji functions in $H$ are of first category in $H$, which strengthens a result proved by $F$. Bagemihl [1].

2. Proof of the Theorem. Letting $\mathfrak{J}$ be the collection of functions in $H$ having a nonempty Tsuji set, we will show that $\mathcal{T}$ is a countable union of sets which are closed and nowhere dense in $H$. If $f \in \mathcal{T}$, then for some $\alpha \in D, x>0$, and $y \in(0,1), L\left(f \circ \phi_{a}, r\right) \leq x$ for all $r \in(y, 1)$. For each triple $(n, m, k)$ of positive integers let $T(n, m, k)$ be the set of functions in $H$ for which there exists $\alpha \in D,|\alpha| \leq 1-1 / n$, such that $L\left(f \circ \phi_{n}, r\right) \leq m$ for all $r \in(1-1 /(k+1), 1)$. It is clear that $\mathcal{T}=\bigcup_{(n, m, k)} T(n, m, k)$, the

Received by the editors April 23, 1974 and, in revised form, June 10, 1974. AMS (MOS) subject classifications (1970). Primary 30A78. 
union being taken over the triples described.

To prove that each $T(n, m, k)$ is closed in $H$, we first state a preparatory lemma.

Lemma 1. Let $\left\{a_{j}\right\}_{j=1}^{\infty}$ be a sequence in $D$ with $\alpha_{j} \rightarrow \alpha \in D$. Let $\phi(z)=$ $(z-\alpha) /(1-\bar{\alpha} z)$, and for each $j, \phi_{j}(z)=\left(z-a_{j}\right) /\left(1-\bar{\alpha}_{j} z\right)$. For a sequence $\left\{f_{j}\right\}_{j=1}^{\infty} \subset H$ with $f_{j} \rightarrow f$ in $H:$

(i) $\phi_{j} \rightarrow \phi$ in $H$,

(ii) $f_{j}^{\circ} \phi_{j} \rightarrow f \circ \phi$ in $H$,

(iii) $\left\{\left(f_{j}^{\circ} \phi_{j}\right)^{*}\right\}_{1}^{\infty}$ converges to $(f \circ \phi)^{*}$ uniformly on compact subsets of $D$,

(iv) for each $r \in(0,1), L\left(f_{j}^{\circ} \phi_{j}, r\right) \rightarrow L(f \circ \phi, r)$.

Lemma 2. Each $T(n, m, k)$ is closed in $H$.

Proof. Let $\left\{f_{j}\right\}$ be a sequence in $T(n, m, k)$ with $f_{j} \rightarrow f$ in $H$. For each $j$ there is a point $\alpha_{j} \in D,\left|a_{j}\right| \leq 1-1 / n$, such that $L\left(f_{j}^{\circ} \phi_{j}, r\right) \leq m$ when $r \in(1-1 /(k+1), 1)$. We may suppose $\alpha_{j} \rightarrow \alpha$, where $|\alpha| \leq 1-1 / n$, and let $\phi(z)=(z-\alpha) /(1-\bar{a} z)$. Lemma 1 (iv) shows that $L(f \circ \phi, r) \leq m$ for each $r \in(1-1 /(k+1), 1)$, so that $f \in T(n, m, k)$.

Lemma 3. Each $T(n, m, k)$ is nowhere dense in $H$.

Proof. For an arbitrary $f \in T(n, m, k)$ we shall show there exists a sequence in $H-T(n, m, k)$ which converges in $H$ to $f$. Since $T(n, m, k)$ is closed, this will show it is nowhere dense in $H$.

For some $\alpha \in D,|\alpha| \leq 1-1 / n, L\left(f \circ \phi_{a}, r\right) \leq m$ for all $r \in(1-1 /(k+1)$, 1). For each positive integer $q$ let $S_{q}$ be the $q$ th partial sum of the Maclaurin's series for $f$.

Given $q$, let $p(q)$ be a positive integer, and define $g_{q}(z)=S_{q}(z)+$ $z^{p(q)}$. As long as $\{p(q)\}_{q=1}^{\infty}$ is increasing, $g_{q} \rightarrow f$ in $H$. If $p(q)$ is sufficiently large, on $C$ both $\left|g_{q}^{\prime}\right|>(q+p(q)) / 2$ and $\left|g_{q}\right| \leq\left|S_{q}\right|+1$. Thus we may take $\rho(q) \epsilon(0,1)$ so that every Jordan curve in the annulus $\rho(q)<|z|<1$ whose interior contains 0 is mapped by $g_{q}$ onto a closed curve of spherical length at least $p(q)$. If $p(q)$ is sufficiently large and $\rho(q)$ is near enough to 1 , we will have $L\left(g_{q} \circ \phi_{a}, r\right)>m$ for a value of $r>1-1 /(k+1)$.

With suitable choice of the sequence $\{p(q)\}_{q=1}^{\infty}$, the sequence $\left\{g_{q}\right\}_{q=1}^{\infty}$ lies in $H-T(n, m, k)$ and converges to $f$ in $H$.

3. In his paper on Tsuji functions [3], W. K. Hayman introduces a larger related class of functions. A function $f \in H$ lies in class $T_{2}$ if 
there exists a sequence $\left\{J_{n}\right\}_{1}^{\infty}$ of Jordan curves in $D$ such that: (i) $J_{n} \subset$ int $J_{n+1}$; (ii) $\min _{J_{n}}|z| \rightarrow 1$ as $n \rightarrow \infty$; (iii) $\lim \sup _{n \rightarrow \infty} \int_{J_{n}} f^{*}(z)|d z|<\infty$. The class $T_{2}$ contains the functions in $H$ with nonempty Tsuji set, so the following question is natural.

Question. Is the class $T_{2}$ of first category in $H$ ?

The author is grateful to the referee for his substantial comments, including a simpler proof of Lemma 3.

\section{REFERENCES}

1. F. Bagemihl, Tsuji points and Tsuji functions, Comment Math. Univ. St. Paul. 17 (1968), 17-20. MR 38 \#6072.

2. E. F. Collingwood and G. Piranian, Tsuji functions with segments of Julia, Math. Z. 84 (1964), 246-253. MR 29 \#3637.

3. W. K. Hayman, The boundary behavior of Tsuji functions, Michigan Math. J. 15 (1968), 1-25. MR 37 \#4265.

4. M. Tsuji, $A$ theorem on the boundary behavior of a meromorphic function in $|z|<1$, Comment Math. Univ. St. Paul. 8 (1960), 53-55. MR 22 \#11131.

DEPARTMENT OF MATHEMATICS, IOWA STATE UNIVERSITY, AMES, IOWA 50010 